\title{
An optimal inventory model for a deteriorating item with time - dependent quadratic demand and delay in payment for two warehouses
}

\author{
P. Muniappan ${ }^{1}$ R. Uthayakumar ${ }^{2}$ and S. Ganesh ${ }^{3}$ \\ ${ }^{1}$ Research Scholar, Sathyabama University, Chennai - 600 119, Tamil Nadu, India. \\ ${ }^{2}$ Associate Professor, Department of Mathematics, Gandhigram Rural Institute - Deemed University, \\ Dindigul- 624 302. Tamil Nadu, India \\ ${ }^{3}$ Head, Sathyabama University, Chennai - 600 119, Tamil Nadu, India. \\ Email: ${ }^{1}$ munichandru@yahoo.com
}

\begin{abstract}
This paper deals with an optimal inventory model for a deteriorating item with time - dependent quadratic demand. When products are seasonal or when supplier provide price discounts for bulk orders, the retailer purchase more items and store it in their warehouses. There are two different types of warehouses. In one, the retailer owns the warehouse, and the other is rented. In this model the retailer first dispose the items in the rented warehouse and then the items present in the own warehouse because of economic reasons. Here permissible delay in payments is allowed. The model is solved analytically to minimize the total inventory cost. The result is illustrated with numerical example for the model.
\end{abstract}

Key words: Inventory, Deteriorating items, Quadratic demand, Delay payments, Credit period, Warehouses

\section{INTRODUCTION}

Deterioration of goods is a common phenomenon in daily life, which is defined as decay, spoilage, damage, evaporation, obsolescence, pilferage, loss of utility. For example sea foods, poultry, pharmaceuticals, chemicals, foodstuff etc., Deteriorating items start deterioration as soon as the retailer receives the commodities. There is another phenomenon called non instantaneous deterioration where most of the deteriorating goods have certain in maintaining quality or the originality. For example fish, fruit, meat, vegetables, alcohols, blood and gasoline. The deteriorating items are stored by retailers in warehouses, when the supplier provides bulk discounts or for seasonal products. The retailer's either having their own warehouse where goods can be stored or when the deteriorating goods are excess they hire a rent warehouse. Generally, the rented warehouse offers many advantages like, better preserving facilities, holding cost etc. From the economic point of view the goods at the rented warehouse is disposed earlier than the own warehouse to reduce the inventory cost.
The past research works emphasize on stock, time, price and inventory level dependent demands. Chung - Yuan Dye (1999) developed an EOQ model for deteriorating items with time varying demand and partial backlogging. Gupta and Vrat (1988) assumed inventory model for stock dependent consumption rate. Mandal and Phaujdar (1989) studied an inventory model for deteriorating items and stock-dependent consumption rate. Shib Sankar Sana (2011) represented pricesensitive demand for perishable items - an EOQ model. Biswajit Sarkar (2012) developed an EOQ model with delay in payments and time varying deterioration rate. Backer and Urban (1986) concentrated on deterministic inventory system with an inventory leveldependent demand rate. Sarker et al. (1997) developed an order - level lot size inventory model with inventory level dependent demand and deterioration. Padmanabhan and Vrat (1995) developed EOQ models for perishable items under stock dependent selling rate.

Sarma (1987) developed a deterministic order level inventory model for deteriorating items with two storage facilities. Pakkala and 
Achary (1992) concentrated on a deterministic inventory model for deteriorating items with two warehouses and finite replenishment rate. Lee (2006) and Niu, Xie (2008) studied two warehouses inventory model with deterioration under FIFO dispatch policy. Chung and Huang developed (2007) the optimal retailer's ordering policies for deteriorating items with limited storage capacity under trade credit financing. Rog et al. (2008) developed a two warehouses inventory model for a deteriorating item with partially/fully backlogged shortage and fuzzy lead time. Dey et al. (2008) proposed two stage inventory problems with dynamic demand and interval valued lead time over finite time horizon under money inflation. A multi warehouse inventory model for items with time varying demand and shortages was developed by Zhou (2003). Lee, Hsu (2009) developed a two warehouse production model for deteriorating inventory items with time dependent demands.

The two trade options available in the market are either the manufacturer receives his amount immediately when the goods are delivered or the credit period given for the buyer to boost the sales thereby to increase profit. Aggarwal and Jaggi (1995) concentrated on ordering policies of deteriorating items under permissible delay in payments. Goyal (1985) developed EOQ model for permissible delay in payments. Hwang and Shrinn (1997) studied exponential deteriorating product under condition of permissible delay in payments. Shinn et al. (1996) developed permissible delay in payments and quantity discount for freight cost. Jamal et al. (1997) considered ordering policy for deteriorating items with shortages and delay in payments. R. Uthayakumar and P. Parvathi (2006) developed a deterministic inventory model for deteriorating items with time dependent demand and partially backlogged shortages with permissible delay in payments. Saoussen Krichen et al. (2011) concentrated on single supplier multiple cooperative retailers inventory model with quantity discount and permissible delay in payments. Yanlai Liang, Fangming Zhou (2011) discussed a two-warehouse inventory model for deteriorating items under conditionally permissible delay in payments.
The detailed description of the paper is as follows. In section 2, assumptions and notations are given. Model formulation is given in section 3. Easily understandable analytical solutions are obtained in these models. In section 4, a numerical example is given in detail to illustrate the models. Finally conclusion and summary are presented.

\section{ASSUMPTIONS AND NOTATIONS}

The following assumptions and notations are used to develop the model

(1) The demand rate $D(t)=a+b t+c t^{2}$, $a \geq 0, b \neq 0, c \neq 0$ at time $\mathrm{t}>0$ is a continuous function of time, where $a, b$ and $\mathrm{c}$ are all constants.

(2) Shortages are not permitted.

(3) Replenishment rate is infinite.

(4) Lead time is zero.

(5) The inventory system considers a single item.

(6) The own warehouse (OW) has limited capacity of w units.

(7) The rented warehouse (RW) has unlimited capacity.

(8) For economic reasons, the items of RW are consumed first and next the items of OW.

(9) The holding cost $\mathrm{h}$ per unit time (excluding interest charges), when $\mathrm{h}=\mathrm{h}_{0}$ for items in $\mathrm{OW}, \mathrm{h}=\mathrm{h}_{\mathrm{r}}$ for items in RW and $\mathrm{h}_{\mathrm{r}}>\mathrm{h}_{0}$

(10) The items deteriorate at a fixed rate $\theta_{1}$ in OW and $\theta_{2}$ in RW, for the rented warehouses offers better facility, so $\theta_{1}>\theta_{2}$ and $h_{r}-h_{0}>p\left(\theta_{1}-\theta_{2}\right)$

(11) To guarantee the optimal solution exists, we assumes that the maximum deteriorating quantity for items in $\mathrm{OW}$, $\theta_{1} w$ is less than the demand rate $\mathrm{D}(\mathrm{t})$, i.e., $\theta_{1} w<D(t)$ 
(12) $r, p_{1}, p$ denote the ordering cost, the sales price per unit and the purchasing cost per unit respectively, where $\mathrm{p}_{1}>\mathrm{p}$. All of these parameters are positive.

(13) The retailer can accumulate revenue and earn interest after his/her customer pays for the amount of purchasing cost to the retailer until the end of the trade credit period by the supplier.

(14) $\mathrm{M}$ is the retailer's trade credit period offered by supplier in years, which is the fraction of the year.

(15) $I_{e}$ denote interest which can be earned per $\$$ per year. $I_{p}$ denotes interest charges per $\$$ in stock per year by the supplier.

(16) $t_{w}$ is the time that inventory level reduce to $\mathrm{w}, \mathrm{T}$ is the length of replenishment cycle.

(17) $\mathrm{I}_{\mathrm{r}}(\mathrm{t})$ denotes the inventory level at time $t \in\left[0, t_{w}\right]$ in RW and $I_{0}(t)$ denotes the level of inventory at time $t \in[0, T]$ in OW

(18) $T C_{i}, \mathrm{i}=1,2,3$ is total cost.

(19) $t_{w}^{*}, T^{*}$ are the optimal solutions.

(20) $T_{c}^{*}$ is the minimum total relevant costs.

\section{MODEL FORMULATION}

The inventory system goes as follows: at time $t=0$, a lot size of certain units enter the system. W units are kept in OW and the rest is stored in RW. The items of OW are consumed only after consuming the goods kept in RW. In the interval $\left[0, t_{w}\right]$, the inventory in $\mathrm{RW}$ gradually decreases due to quadratic demand and deterioration and it vanishes at $t=t_{w}$. In OW, however, the inventory $\mathrm{w}$ decreases during $\left[0, t_{w}\right]$ due to deterioration only, but during $\left[t_{w}, T\right]$, the inventory is depleted due to both demand and deterioration. By the time to $\mathrm{T}$, both warehouses are empty.
At time $t \in\left[0, t_{w}\right]$, the inventory level in RW and $\mathrm{OW}$ is given by the following differential equation

$\frac{d I_{r}(t)}{d t}=-a-b t-c t^{2}-\theta_{2} I_{r}(t), 0 \leq t \leq t_{w}$, with the boundary condition $I_{r}\left(t_{w}\right)=0$ and

$\frac{d I_{o}(t)}{d t}=-\theta_{1} I_{o}(t), 0 \leq t \leq t_{w}$, with the initial condition $I_{0}(0)=0$.

At time $t \in\left[t_{w}, T\right]$, the inventory level in OW is given by the following differential equation

$\frac{d I_{o}(t)}{d t}=-a-b t-c t^{2}-\theta_{1} I_{o}(t), t_{w} \leq t \leq T$, with the boundary condition $I_{o}(T)=0$.

Solving the above differential equations, we get

$$
\begin{gathered}
I_{r}(t)=\frac{1}{\theta^{2}}\left[\left(a+b t_{w}+c t_{w}^{2}\right) e^{\theta_{2}\left(t_{w}-t\right)}-(a+b t+\right. \\
\left.\left.c t^{2}\right)\right]+\frac{1}{\theta_{2}^{2}}\left[(b+2 c t)-\left(b+2 c t_{w}\right) e^{\theta_{2}\left(t_{w}-t\right)}\right]+ \\
\frac{2 c}{\theta_{2}^{3}}\left[e^{\theta_{2}\left(t_{w}-t\right)}-1\right], 0 \leq t \leq t_{w} \\
I_{0}(t)=w e^{\theta_{1} t}, 0 \leq t \leq t_{w}
\end{gathered}
$$

and

$$
\begin{aligned}
I_{o}(t)= & \frac{1}{\theta_{1}}\left[\left(a+b T+c T^{2}\right) e^{\theta_{1}(T-t)}-\left(a+b t+c t^{2}\right)\right]+ \\
& \frac{1}{\theta_{1}^{2}}\left[(b+2 c t)-(b+2 c T) e^{\theta_{1}(T-t)}\right]+ \\
& \frac{2 c}{\theta_{1}^{3}}\left[e^{\theta_{1}(T-t)}-1\right], t_{w} \leq t \leq T
\end{aligned}
$$

Considering the continuity of $I_{o}(t)$ at time $t=t_{w}$,

i.e., $I_{0}\left(t_{w}\right)=w e^{\theta_{1} t_{w}}$

Now the annual total cost per cycle is comprised of the following parts:

(i) Annual ordering cost $\frac{r}{T}$

(ii) Holding Cost

The annual holding cost in RW during the interval $\left[0, t_{w}\right]$ is

$\frac{h_{r}}{T} \int_{0}^{t_{w}} I_{r}(t) d t$ 


$$
\begin{aligned}
= & \frac{h_{r}}{T}\left\{\frac { 1 - e ^ { \theta _ { 2 } ( t _ { w } - t ) } } { \theta _ { 2 } ^ { 4 } } \left[\theta_{2}^{2}\left(a+b t_{w}+c t_{w}^{2}\right)-\right.\right. \\
& \left.\theta_{2}\left(b+2 c t_{w}\right)+2 c\right]+\frac{1}{\theta_{2}^{3}}\left[\theta_{2}\left(b t_{w}+c t_{w}^{2}\right)-\right. \\
& \left.\left.\theta_{2}^{2}\left(a t_{w}+\frac{b t_{w}^{2}}{2}+\frac{c t_{w}^{3}}{3}\right)-2 c t_{w}\right]\right\}
\end{aligned}
$$

And the annual holding cost in OW during the interval $[0, T]$ is

$$
\begin{aligned}
& \frac{h_{o}}{T} \int_{0}^{T} I_{o}(t) d t \\
= & \frac{h_{o}}{T}\left[\int_{0}^{t_{w}} I_{o}(t) d t+\int_{t_{w}}^{T} I_{o}(t) d t\right] \\
= & \frac{h_{o}}{T}\left\{\frac{-w}{\theta_{1}}\left(e^{-\theta_{1} t_{w}}-1\right)-\frac{1}{\theta_{1}^{4}}\left[\theta_{1}^{2}(a+b T+\right.\right. \\
& \left.\left.c T^{2}\right)-\theta_{1}(b+2 c T)+2 c\right]\left[1-e^{\theta_{1}\left(T-t_{w}\right)}\right]+ \\
& \frac{1}{\theta_{1}^{3}}\left[\theta_{1}\left(b T+c T^{2}\right)-\theta_{1}^{2}\left(a T+\frac{b T^{2}}{2}+\frac{c T^{3}}{3}\right)-\right. \\
& 2 c T-\theta_{1}\left(b t_{w}+c t_{w}^{2}\right)+\theta_{1}^{2}\left(a t_{w}+\frac{b t_{w}^{2}}{2}+\right. \\
& \left.\left.\left.\frac{c t_{w}^{3}}{3}\right)+2 c t_{w}\right]\right\}
\end{aligned}
$$

(iii) Deterioration Cost

The amount of deteriorated items in both RW and OW during $[0, T]$ is

$$
\begin{aligned}
\frac{P}{T} & {\left[\theta_{2} \int_{0}^{t_{w}} I_{o}(t) d t+\theta_{1} \int_{t_{w}}^{T} I_{o}(t) d t\right] } \\
= & \frac{P}{T}\left[\theta _ { 2 } \left\{\frac { 1 - e ^ { \theta _ { 2 } ( t _ { w } - t ) } } { \theta _ { 2 } ^ { 4 } } \left[\theta_{2}^{2}\left(a+b t_{w}+c t_{w}^{2}\right)-\right.\right.\right. \\
& \left.\theta_{2}\left(b+2 c t_{w}\right)+2 c\right]+\frac{1}{\theta_{2}^{3}}\left[\theta_{2}\left(b t_{w}+c t_{w}^{2}\right)-\right. \\
& \left.\left.\theta_{2}^{2}\left(a t_{w}+\frac{b t_{w}^{2}}{2}+\frac{c t_{w}^{3}}{3}\right)-2 c t_{w}\right]\right\}+ \\
& \theta_{1}\left\{\frac{-w}{\theta_{1}}\left(e^{-\theta_{1} t_{w}}-1\right)-\frac{1}{\theta_{1}^{4}}\left[\theta_{1}^{2}\left(a+b T+c T^{2}\right)-\right.\right. \\
& \left.\theta_{1}(b+2 c T)+2 c\right]\left[1-e^{\theta_{1}\left(T-t_{w}\right)}\right]+ \\
& \frac{1}{\theta_{1}^{3}}\left[\theta_{1}\left(b T+c T^{2}\right)-\theta_{1}^{2}\left(a T+\frac{b T^{2}}{2}+\frac{c T^{3}}{3}\right)-\right. \\
& 2 c T-\theta_{1}\left(b t_{w}+c t_{w}^{2}\right)+\theta_{1}^{2}\left(a t_{w}+\frac{b t_{w}^{2}}{2}+\frac{c t_{w}^{3}}{3}\right)+ \\
& \left.\left.\left.2 c t_{w}\right]\right\}\right]
\end{aligned}
$$

(iv) Interest charged

There are three cases

Case 1: $M \leq t_{w} \leq T$

In this case, the annual interest payable is

$$
\frac{P I_{p}}{T}\left\{\int_{M}^{t_{w}} I_{r}(t) d t+\int_{M}^{t_{w}} I_{o}(t) d t+\int_{t_{w}}^{T} I_{o}(t) d t\right\}
$$

$$
\begin{aligned}
= & \frac{P I_{p}}{T}\left\{\frac { e ^ { \theta _ { 2 } ( t _ { w } - M ) } - 1 } { \theta _ { 2 } ^ { 4 } } \left[\theta_{2}^{2}\left(a+b t_{w}+c t_{w}^{2}\right)-\theta_{2}\left(b+2 c t_{w}\right)+\right.\right. \\
& 2 c]+\frac{1}{\theta_{2}^{3}}\left[\theta_{2}\left[b\left(t_{w}-M\right)+c\left(t_{w}^{2}-M^{2}\right)\right]+\right. \\
& \theta_{2}^{2}\left(a\left(M-t_{w}\right)+\frac{b\left(M^{2}-t_{w}^{2}\right)}{2}+\frac{c\left(M^{3}-t_{w}^{3}\right)}{3}\right)+ \\
& \left.2 c\left(M-t_{w}\right)\right]+\frac{w}{\theta_{1}}\left[e^{-\theta_{1} M}-e^{-\theta_{1} t_{w}}\right]+ \\
& \frac{1}{\theta_{1}}\left[\left(\frac{a+b T+c T^{2}}{\theta_{1}}\right)\left(e^{\theta_{1}\left(T-t_{w}\right)}-1\right)+a\left(t_{w}-T\right)+\right. \\
& \left.\frac{b\left(t_{w}^{2}-T^{2}\right)}{2}+\frac{c\left(t_{w}^{3}-T^{3}\right)}{3}\right]+\frac{1}{\theta_{1}^{2}}\left[\left[b\left(T-t_{w}\right)+c\left(T^{2}-t_{w}^{2}\right)\right]+\right. \\
& \left.\left(\frac{b+2 c T}{\theta_{1}}\right)\left(1-e^{\theta_{1}\left(T-t_{w}\right)}\right)\right]+\frac{2 c}{\theta_{1}^{3}}\left[\frac{e^{\theta_{1}\left(T-t_{w}\right)}-1}{\theta_{1}}+\left(t_{w}-\right.\right. \\
& T)]\}
\end{aligned}
$$

Case 2: $t_{w} \leq M \leq T$

In this case, the annual interest payable is

$$
\frac{P I_{p}}{T} \int_{M}^{T} I_{0}(t) d t
$$$$
=\frac{P I_{p}}{T}\left\{\frac { 1 } { \theta _ { 1 } } \left[\left(\frac{a+b T+c T^{2}}{\theta_{1}}\right)\left(e^{\theta_{1}(T-M)}-1\right)+a(M-\right.\right.
$$$$
\left.T)+\frac{b\left(M^{2}-T^{2}\right)}{2}+\frac{c\left(M^{3}-T^{3}\right)}{3}\right]+\frac{1}{\theta_{1}^{2}}\left[[b T-M)+c\left(T^{2}-\right.\right.
$$$$
\left.\left.\left.M^{2}\right)\right]+\left(\frac{b+2 c T}{\theta_{1}}\right)\left(1-e^{\theta_{1}(T-M)}\right)\right]+
$$$$
\left.\frac{2 c}{\theta_{1}^{3}}\left[\frac{1}{\theta_{1}}\left(e^{\theta_{1}(T-M)}-1\right)+(M-T)\right]\right\}
$$

Case 3: $\mathrm{M}>\mathrm{T}$

In this case, no interest charges are paid for the items.

(v) Interest earned

There are two cases

Case1: $M \leq T$

In this annual interest earned is

$\frac{p_{1} I_{e}}{T} \int_{0}^{M} D(t) t d t=\frac{p_{1} I_{e} M^{2}}{12 T}[6 a+4 b+3 c]$

\section{Case2: $\mathrm{M}>\mathrm{T}$}

In this case, the annual interest earned is

$\frac{p_{1} I_{e}}{T}\left\{\int_{0}^{T} D(t) t d t+T(M-T) D(t)\right\}$ 


$$
\begin{aligned}
= & p_{1} I_{e}\left\{\frac{T}{12}[6 a+4 b+3 c]+(M-T)(a+\right. \\
& \left.\left.b T+c T^{2}\right)\right\}
\end{aligned}
$$

Therefore the total cost can be formulated as

$$
T C\left(t_{w}, T\right)= \begin{cases}T C_{1}, & M \leq t_{w} \leq T \\ T C_{2}, & t_{w} \leq M \leq T \\ T C_{3}, & M>T\end{cases}
$$

where

$$
\begin{aligned}
& T C_{1}=\frac{1}{T}\left\{r+\left(h_{r}+P \theta_{2}\right)\left\{\frac { 1 - e ^ { \theta _ { 2 } ( t _ { w } - t ) } } { \theta _ { 2 } ^ { 4 } } \left[\theta_{2}^{2}(a+\right.\right.\right. \\
& \left.\left.b t_{w}+c t_{w}^{2}\right)-\theta_{2}\left(b+2 c t_{w}\right)+2 c\right]+\frac{1}{\theta_{2}^{3}}\left[\theta _ { 2 } \left(b t_{w}+\right.\right. \\
& \left.\left.\left.c t_{w}^{2}\right)-\theta_{2}^{2}\left(a t_{w}+\frac{b t_{w}^{2}}{2}+\frac{c t_{w}^{3}}{3}\right)-2 c t_{w}\right]\right\}+ \\
& \left(h_{0}+P \theta_{1}\right)\left\{\frac{w}{\theta_{1}}\left(1-e^{-\theta_{1} t_{w}}\right)-\frac{1-e^{\theta_{1}\left(T-t_{w}\right)}}{\theta_{1}^{4}}\left[\theta_{1}^{2}(a+\right.\right. \\
& \left.\left.b T+c T^{2}\right)-\theta_{1}(b+2 c T)+2 c\right]+\frac{1}{\theta_{1}^{3}}\left[\theta_{1}[b(T-\right. \\
& \left.\left.t_{w}\right)+c\left(T^{2}-t_{w}^{2}\right)\right]-\theta_{1}^{2}\left(a\left(t_{w}-T\right)+\frac{b\left(t_{w}^{2}-T^{2}\right)}{2}+\right. \\
& \left.\left.\left.\frac{c\left(t_{w}^{3}-T^{3}\right)}{3}\right)+2 c\left(t_{w}-T\right)\right]\right\}+ \\
& \mathrm{PI}_{\mathrm{p}}\left\{\frac { e ^ { \theta _ { 2 } ( t _ { w } - M ) } - 1 } { \theta _ { 2 } ^ { 4 } } \left[\theta_{2}^{2}\left(a+b t_{w}+c t_{w}^{2}\right)-\right.\right. \\
& \left.\theta_{2}\left(b+2 c t_{w}\right)+2 c\right]+\frac{1}{\theta_{2}^{3}}\left[\theta _ { 2 } \left[b\left(t_{w}-M\right)+c\left(t_{w}^{2}-\right.\right.\right. \\
& \left.\left.M^{2}\right)\right]+\theta_{2}^{2}\left(a\left(M-t_{w}\right)+\frac{b\left(M^{2}-t_{w}^{2}\right)}{2}+\frac{c\left(M^{3}-t_{w}^{3}\right)}{3}\right)+ \\
& \left.2 c\left(M-t_{w}\right)\right]+\frac{w}{\theta_{1}}\left[e^{-\theta_{1} M}-e^{-\theta_{1} t_{w}}\right]+ \\
& \frac{1}{\theta_{1}}\left[\left(\frac{a+b T+c T^{2}}{\theta_{1}}\right)\left(e^{\theta_{1}\left(T-t_{w}\right)}-1\right)+a\left(t_{w}-T\right)+\right. \\
& \left.\frac{b\left(t_{w}^{2}-T^{2}\right)}{2}+\frac{c\left(t_{w}^{3}-T^{3}\right)}{3}\right]+\frac{1}{\theta_{1}^{2}}\left[\left[b\left(T-t_{w}\right)+c\left(T^{2}-\right.\right.\right. \\
& \left.\left.\left.t_{w}^{2}\right)\right]+\left(\frac{b+2 c T}{\theta_{1}}\right)\left(1-e^{\theta_{1}\left(T-t_{w}\right)}\right)\right]+\frac{2 c}{\theta_{1}^{3}}\left[\frac{e^{\theta_{1}\left(T-t_{w}\right)}-1}{\theta_{1}}+\right. \\
& \left.\left.\left.\left(t_{w}-T\right)\right]\right\}-\frac{p_{1} I_{e} M^{2}}{12}[6 a+4 b+3 c]\right\}
\end{aligned}
$$

$T C_{2}=\frac{1}{T}\left\{r+\left(h_{r}+P \theta_{2}\right)\left\{\frac{1-e^{\theta_{2}\left(t_{w}-t\right)}}{\theta_{2}^{4}}\left[\theta_{2}^{2}(a+\right.\right.\right.$ $\left.\left.b t_{w}+c t_{w}^{2}\right)-\theta_{2}\left(b+2 c t_{w}\right)+2 c\right]+\frac{1}{\theta_{2}^{3}}\left[\theta_{2}\left(b t_{w}+\right.\right.$ $\left.\left.\left.c t_{w}^{2}\right)-\theta_{2}^{2}\left(a t_{w}+\frac{b t_{w}^{2}}{2}+\frac{c t_{w}^{3}}{3}\right)-2 c t_{w}\right]\right\}+$ $\left(h_{0}+P \theta_{1}\right)\left\{\frac{w}{\theta_{1}}\left(1-e^{-\theta_{1} t_{w}}\right)-\frac{1-e^{\theta_{1}\left(T-t_{w}\right)}}{\theta_{1}^{4}}\left[\theta_{1}^{2}(a+\right.\right.$ $\left.\left.b T+c T^{2}\right)-\theta_{1}(b+2 c T)+2 c\right]+\frac{1}{\theta_{1}^{3}}\left[\theta_{1}[b(T-\right.$ $\left.\left.t_{w}\right)+c\left(T^{2}-t_{w}^{2}\right)\right]-\theta_{1}^{2}\left(a\left(t_{w}-T\right)+\frac{b\left(t_{w}^{2}-T^{2}\right)}{2}+\right.$ $\left.\left.\left.\frac{c\left(t_{w}^{3}-T^{3}\right)}{3}\right)+2 c\left(t_{w}-T\right)\right]\right\}+$ $\mathrm{PI}_{\mathrm{p}}\left\{\frac{1}{\theta_{1}}\left[\left(\frac{a+b T+c T^{2}}{\theta_{1}}\right)\left(e^{\theta_{1}(T-M)}-1\right)+a(M-T)+\right.\right.$
$\left.\frac{b\left(M^{2}-T^{2}\right)}{2}+\frac{c\left(M^{3}-T^{3}\right)}{3}\right]+\frac{1}{\theta_{1}^{2}}\left[[b T-M)+c\left(T^{2}-\right.\right.$

$\left.\left.\left.M^{2}\right)\right]+\left(\frac{b+2 c T}{\theta_{1}}\right)\left(1-e^{\theta_{1}(T-M)}\right)\right]+\frac{2 c}{\theta_{1}^{3}}\left[\frac{e^{\theta_{1}(T-M)}-1}{\theta_{1}}+\right.$

$\left.(M-T)]\}-\frac{p_{1} I_{e} M^{2}}{12}[6 a+4 b+3 c]\right\}$

$T C_{3}=\frac{1}{T}\left\{r+\left(h_{r}+P \theta_{2}\right)\left\{\frac{1-e^{\theta_{2}\left(t_{w}-t\right)}}{\theta_{2}^{4}}\left[\theta_{2}^{2}(a+\right.\right.\right.$

$\left.\left.b t_{w}+c t_{w}^{2}\right)-\theta_{2}\left(b+2 c t_{w}\right)+2 c\right]+\frac{1}{\theta_{2}^{3}}\left[\theta_{2}\left(b t_{w}+\right.\right.$

$\left.\left.\left.c t_{w}^{2}\right)-\theta_{2}^{2}\left(a t_{w}+\frac{b t_{w}^{2}}{2}+\frac{c t_{w}^{3}}{3}\right)-2 c t_{w}\right]\right\}+$

$\left(h_{0}+P \theta_{1}\right)\left\{\frac{w}{\theta_{1}}\left(1-e^{-\theta_{1} t_{w}}\right)-\frac{1-e^{\theta_{1}\left(T-t_{w}\right)}}{\theta_{1}^{4}}\left[\theta_{1}^{2}(a+\right.\right.$

$\left.\left.b T+c T^{2}\right)-\theta_{1}(b+2 c T)+2 c\right]+\frac{1}{\theta_{1}^{3}}\left[\theta_{1}[b(T-\right.$

$\left.\left.t_{w}\right)+c\left(T^{2}-t_{w}^{2}\right)\right]-\theta_{1}^{2}\left(a\left(t_{w}-T\right)+\frac{b\left(t_{w}^{2}-T^{2}\right)}{2}+\right.$

$\left.\left.\left.\frac{c\left(t_{w}^{3}-T^{3}\right)}{3}\right)+2 c\left(t_{w}-T\right)\right]\right\}+$

$\mathrm{PI}_{\mathrm{p}}\left\{\frac{1}{\theta_{1}}\left[\left(\frac{a+b T+c T^{2}}{\theta_{1}}\right)\left(e^{\theta_{1}(T-M)}-1\right)+a(M-T)+\right.\right.$

$\left.\frac{b\left(M^{2}-T^{2}\right)}{2}+\frac{c\left(M^{3}-T^{3}\right)}{3}\right]+\frac{1}{\theta_{1}^{2}}[b T-M)+c\left(T^{2}-\right.$

$\left.\left.\left.M^{2}\right)\right]+\left(\frac{b+2 c T}{\theta_{1}}\right)\left(1-e^{\theta_{1}(T-M)}\right)\right]+\frac{2 c}{\theta_{1}^{3}}\left[\frac{e^{\theta_{1}(T-M)}-1}{\theta_{1}}+\right.$

$(M-T)]\}-p_{1} I_{e}\left\{\frac{T^{2}(6 a+4 b+3 c)}{12}+T(M-T)(a+\right.$

$\left.\left.\left.b T+c T^{2}\right)\right\}\right\}$

\section{Case 1: $M \leq t_{w} \leq T$}

For optimality (i.e., to find optimal $T^{*}$ and $t_{w}^{*}$ ) we have to solve

$$
\frac{\partial T C_{1}\left(t_{w}, T\right)}{\partial t_{w}}=0 \text { and } \quad \frac{\partial T C_{1}\left(t_{w}, T\right)}{\partial T}=0
$$

Provided they satisfy the sufficient conditions

$\left[\frac{\partial^{2} T C_{1}\left(t_{w}, T\right)}{\partial t_{w}^{2}}\right]_{a t\left(t_{w}^{*}, T^{*}\right)}>0,\left[\frac{\partial^{2} T C_{1}\left(t_{w}, T\right)}{\partial T^{2}}\right]_{a t\left(t_{w}^{*}, T^{*}\right)}>0$

and $\left[\left(\frac{\partial^{2} T C_{1}\left(t_{w}, T\right)}{\partial t_{w}^{2}}\right)\left(\frac{\partial^{2} T C_{1}\left(t_{w}, T\right)}{\partial T^{2}}\right)-\left(\frac{\partial^{2} T C_{1}\left(T_{1}, T\right)}{\partial t_{w} \partial T}\right)^{2}\right]>0$

Now we shall solve

$\frac{\partial T C_{1}\left(t_{w}, T\right)}{\partial t_{w}}=0$ and $\frac{\partial T C_{1}\left(t_{w}, T\right)}{\partial T}=0$ to get the optimal values of $T^{*}$ and $t_{w}^{*}$

$\frac{\partial T C_{1}\left(t_{w}, T\right)}{\partial t_{w}}=0$ 


$$
\begin{aligned}
& \Rightarrow \frac{1}{T}\left\{\left(h_{r}+P \theta_{2}\right)\left(\frac{a+b t_{w}+c t_{w}^{2}}{\theta_{2}}\right)\left(e^{\theta_{2} t_{w}}-1\right)+\right. \\
& \left(h_{0}+P \theta_{1}\right)\left\{w e^{\theta_{1} t_{w}}-\frac{e^{\theta_{1}\left(t_{w}-t\right)}}{\theta_{1}^{3}}\left[\theta_{1}^{2}(a+b T+\right.\right. \\
& \left.\left.c T^{2}\right)-\theta_{1}(b+2 c)+2 c\right]+\frac{1}{\theta_{1}^{3}}\left[-\theta_{1}(b+c)+\right. \\
& \left.\left.\theta_{1}^{2}\left(a+b t_{w}+c t_{w}^{2}\right)+2 c\right]\right\}+ \\
& P I_{p}\left\{\frac{a+b t_{w}+c t_{w}^{2}}{\theta_{2}}\left(e^{\theta_{2}\left(t_{w}-M\right)}-1\right)+w e^{\theta_{1} t_{w}}+\right. \\
& \frac{1}{\theta_{1}}\left[e^{\theta_{1}\left(t_{w}-M\right)}\left(a+b T+c T^{2}\right)+a+b t_{w}+c t_{w}^{2}\right]+ \\
& \frac{1}{\theta_{1}^{2}}\left[-\left(b+2 c t_{w}\right)+(b+2 c T) e^{\theta_{1}\left(T-t_{w}\right)}\right]+ \\
& \left.\left.\frac{2 c}{\theta_{1}^{3}}\left(e^{\theta_{1}\left(T-t_{w}\right)}+1\right)\right\}\right\} \\
& \frac{\partial T C_{1}\left(t_{w}, T\right)}{\partial T}=0 \\
& \Rightarrow \frac{1}{T}\left\{\left(h_{0}+P \theta_{1}\right)\left\{\frac{a+b T+c T^{2}}{\theta_{1}} e^{\theta_{1}\left(T-t_{w}\right)}-\frac{b+2 c T}{\theta_{1}^{2}}+\frac{2 c}{\theta_{1}^{3}}\right\}+\right. \\
& \left.P I_{p}\left\{\frac{a+b T+c T^{2}}{\theta_{1}}\left(e^{\theta_{1}\left(T-t_{w}\right)}-1\right)\right\}\right\}-\frac{1}{T^{2}}\left\{r+\left(h_{r}+\right.\right. \\
& \left.P \theta_{2}\right)\left\{\frac { 1 - e ^ { \theta _ { 2 } ( t _ { w } - t ) } } { \theta _ { 2 } ^ { 4 } } \left[\theta_{2}^{2}\left(a+b t_{w}+c t_{w}^{2}\right)-\theta_{2}\left(b+2 c t_{w}\right)+\right.\right. \\
& 2 c]+\frac{1}{\theta_{2}^{3}}\left[\theta_{2}\left(b t_{w}+c t_{w}^{2}\right)-\theta_{2}^{2}\left(a t_{w}+\frac{b t_{w}^{2}}{2}+\frac{c t_{w}^{3}}{3}\right)-\right. \\
& \left.\left.2 c t_{w}\right]\right\}+ \\
& \left(h_{0}+P \theta_{1}\right)\left\{\frac{w}{\theta_{1}}\left(1-e^{-\theta_{1} t_{w}}\right)-\frac{1-e^{\theta_{1}\left(T-t_{w}\right)}}{\theta_{1}^{4}}\left[\theta_{1}^{2}(a+b T+\right.\right. \\
& \left.\left.c T^{2}\right)-\theta_{1}(b+2 c T)+2 c\right]+\frac{1}{\theta_{1}^{3}}\left[\theta _ { 1 } \left[b\left(T-t_{w}\right)+c\left(T^{2}-\right.\right.\right. \\
& \left.\left.t_{w}^{2}\right)\right]-\theta_{1}^{2}\left(a\left(t_{w}-T\right)+\frac{b\left(t_{w}^{2}-T^{2}\right)}{2}+\frac{c\left(t_{w}^{3}-T^{3}\right)}{3}\right)+2 c\left(t_{w}-\right. \\
& T)]\}+\mathrm{PI}_{\mathrm{p}}\left\{\frac { e ^ { \theta _ { 2 } ( t _ { w } - M ) } - 1 } { \theta _ { 2 } ^ { 4 } } \left[\theta_{2}^{2}\left(a+b t_{w}+c t_{w}^{2}\right)-\right.\right. \\
& \left.\theta_{2}\left(b+2 c t_{w}\right)+2 c\right]+\frac{1}{\theta_{2}^{3}}\left[\theta _ { 2 } \left[b\left(t_{w}-M\right)+c\left(t_{w}^{2}-\right.\right.\right. \\
& \left.\left.M^{2}\right)\right]+\theta_{2}^{2}\left(a\left(M-t_{w}\right)+\frac{b\left(M^{2}-t_{w}^{2}\right)}{2}+\frac{c\left(M^{3}-t_{w}^{3}\right)}{3}\right)+ \\
& \left.2 c\left(M-t_{w}\right)\right]+\frac{w}{\theta_{1}}\left[e^{-\theta_{1} M}-e^{-\theta_{1} t_{w}}\right]+ \\
& \frac{1}{\theta_{1}}\left[\left(\frac{a+b T+c T^{2}}{\theta_{1}}\right)\left(e^{\theta_{1}\left(T-t_{w}\right)}-1\right)+a\left(t_{w}-T\right)+\frac{b\left(t_{w}^{2}-T^{2}\right)}{2}+\right. \\
& \left.\frac{c\left(t_{w}^{3}-T^{3}\right)}{3}\right]+\frac{1}{\theta_{1}^{2}}\left[\left[b\left(T-t_{w}\right)+c\left(T^{2}-t_{w}^{2}\right)\right]+\right. \\
& \left.\left(\frac{b+2 c T}{\theta_{1}}\right)\left(1-e^{\theta_{1}\left(T-t_{w}\right)}\right)\right]+\frac{2 c}{\theta_{1}^{3}}\left[\frac{e^{\theta_{1}\left(T-t_{w}\right)}-1}{\theta_{1}}+\left(t_{w}-\right.\right. \\
& \left.T)]\}-\frac{p_{1} I_{e} M^{2}}{12}[6 a+4 b+3 c]\right\}
\end{aligned}
$$

\section{Case 2: $t_{w} \leq M \leq T$}

For optimality (i.e., to find optimal $T^{*}$ and $t_{w}^{*}$ ) we have to solve,

$\frac{\partial T C_{2}\left(t_{w}, T\right)}{\partial t_{w}}=0 \quad$ and $\quad \frac{\partial T C_{2}\left(t_{w}, T\right)}{\partial T}=0$

provided they satisfy the sufficient conditions

$$
\begin{aligned}
& {\left[\frac{\partial^{2} T C_{2}\left(t_{w}, T\right)}{\partial t_{w}^{2}}\right]_{a t\left(t_{w}^{*}, T^{*}\right)}>0,\left[\frac{\partial^{2} T C_{2}\left(t_{w}, T\right)}{\partial T^{2}}\right]_{a t\left(t_{w}^{*}, T^{*}\right)}>0} \\
& \text { and }\left[\left(\frac{\partial^{2} T C_{2}\left(t_{w}, T\right)}{\partial t_{w}^{2}}\right)\left(\frac{\partial^{2} T C_{2}\left(t_{w}, T\right)}{\partial T^{2}}\right)-\left(\frac{\partial^{2} T C_{2}\left(T_{1}, T\right)}{\partial t_{w} \partial T}\right)^{2}\right]>0
\end{aligned}
$$

Now we shall solve

$\frac{\partial T C_{2}\left(t_{w}, T\right)}{\partial t_{w}}=0$ and $\frac{\partial T C_{2}\left(t_{w}, T\right)}{\partial T}=0$ to get the optimal values of $T^{*}$ and $t_{w}^{*}$

$\frac{\partial T C_{2}\left(t_{w}, T\right)}{\partial t_{w}}=0$

$\Rightarrow \frac{1}{T}\left\{\left(h_{r}+P \theta_{2}\right)\left(\frac{a+b t_{w}+c t_{w}^{2}}{\theta_{2}}\right)\left(e^{\theta_{2} t_{w}}-1\right)+\right.$

$\left(h_{0}+P \theta_{1}\right)\left\{w e^{\theta_{1} t_{w}}-\frac{e^{\theta_{1}\left(t_{w}-t\right)}}{\theta_{1}^{3}}\left[\theta_{1}^{2}(a+b T+\right.\right.$

$\left.\left.c T^{2}\right)-\theta_{1}(b+2 c)+2 c\right]+\frac{1}{\theta_{1}^{3}}\left[-\theta_{1}(b+c)+\right.$

$\left.\left.\left.\theta_{1}^{2}\left(a+b t_{w}+c t_{w}^{2}\right)+2 c\right]\right\}\right\}$

$\frac{\partial T C_{2}\left(t_{w}, T\right)}{\partial T}=0$

$\Rightarrow \frac{1}{T}\left\{\left(h_{0}+P \theta_{1}\right)\left\{\frac{a+b T+c T^{2}}{\theta_{1}} e^{\theta_{1}\left(T-t_{w}\right)}-\frac{b+2 c T}{\theta_{1}^{2}}+\frac{2 c}{\theta_{1}^{3}}\right\}+\right.$

$\left.P I_{p}\left\{\frac{a+b T+c T^{2}}{\theta_{1}}\left(e^{\theta_{1}(T-M)}-1\right)\right\}\right\}-\frac{1}{T^{2}}\left\{r+\left(h_{r}+\right.\right.$

$\left.P \theta_{2}\right)\left\{\frac{1-e^{\theta_{2}\left(t_{w}-t\right)}}{\theta_{2}^{4}}\left[\theta_{2}^{2}\left(a+b t_{w}+c t_{w}^{2}\right)-\theta_{2}\left(b+2 c t_{w}\right)+\right.\right.$

$2 c]+\frac{1}{\theta_{2}^{3}}\left[\theta_{2}\left(b t_{w}+c t_{w}^{2}\right)-\theta_{2}^{2}\left(a t_{w}+\frac{b t_{w}^{2}}{2}+\frac{c t_{w}^{3}}{3}\right)-\right.$

$\left.\left.2 c t_{w}\right]\right\}+$

$\left(h_{0}+P \theta_{1}\right)\left\{\frac{w}{\theta_{1}}\left(1-e^{-\theta_{1} t_{w}}\right)-\frac{1-e^{\theta_{1}\left(T-t_{w}\right)}}{\theta_{1}^{4}}\left[\theta_{1}^{2}(a+b T+\right.\right.$

$\left.\left.c T^{2}\right)-\theta_{1}(b+2 c T)+2 c\right]+\frac{1}{\theta_{1}^{3}}\left[\theta_{1}\left[b\left(T-t_{w}\right)+c\left(T^{2}-\right.\right.\right.$

$\left.\left.t_{w}^{2}\right)\right]-\theta_{1}^{2}\left(a\left(t_{w}-T\right)+\frac{b\left(t_{w}^{2}-T^{2}\right)}{2}+\frac{c\left(t_{w}^{3}-T^{3}\right)}{3}\right)+2 c\left(t_{w}-\right.$

$T)]\}+\mathrm{PI}_{\mathrm{p}}\left\{\frac{1}{\theta_{1}}\left[\left(\frac{a+b T+c T^{2}}{\theta_{1}}\right)\left(e^{\theta_{1}(T-M)}-1\right)+a(M-\right.\right.$

$\left.T)+\frac{b\left(M^{2}-T^{2}\right)}{2}+\frac{c\left(M^{3}-T^{3}\right)}{3}\right]+\frac{1}{\theta_{1}^{2}}\left[[b T-M)+c\left(T^{2}-\right.\right.$

$\left.\left.\left.M^{2}\right)\right]+\left(\frac{b+2 c T}{\theta_{1}}\right)\left(1-e^{\theta_{1}(T-M)}\right)\right]+\frac{2 c}{\theta_{1}^{3}}\left[\frac{e^{\theta_{1}(T-M)}-1}{\theta_{1}}+(M-\right.$

$\left.T)]\}-\frac{p_{1} I_{e} M^{2}}{12}[6 a+4 b+3 c]\right\}$

\section{Case 3: $M>T$}

For optimality (i.e., to find optimal $T^{*}$ and $t_{w}^{*}$ ) we have to solve,

$\frac{\partial T C_{3}\left(t_{w}, T\right)}{\partial t_{w}}=0 \quad$ and $\quad \frac{\partial T C_{3}\left(t_{w}, T\right)}{\partial T}=0$

provided they satisfy the sufficient conditions 


$$
\begin{aligned}
& {\left[\frac{\partial^{2} T C_{3}\left(t_{w}, T\right)}{\partial t_{w}^{2}}\right]_{a t\left(t_{w}^{*}, T^{*}\right)}>0,\left[\frac{\partial^{2} T C_{3}\left(t_{w}, T\right)}{\partial T^{2}}\right]_{a t\left(t_{w}^{*}, T^{*}\right)}>0} \\
& \text { and }\left[\left(\frac{\partial^{2} T C_{3}\left(t_{w}, T\right)}{\partial t_{w}^{2}}\right)\left(\frac{\partial^{2} T C_{3}\left(t_{w}, T\right)}{\partial T^{2}}\right)-\left(\frac{\partial^{2} T C_{3}\left(T_{1}, T\right)}{\partial t_{w} \partial T}\right)^{2}\right]>0
\end{aligned}
$$

Now we shall solve

$\frac{\partial T C_{3}\left(t_{w}, T\right)}{\partial t_{w}}=0$ and $\frac{\partial T C_{3}\left(t_{w}, T\right)}{\partial T}=0$ to get the optimal values of $T^{*}$ and $t_{w}^{*}$

$\frac{\partial T C_{3}\left(t_{w}, T\right)}{\partial t_{w}}=0$

$\Rightarrow \frac{1}{T}\left\{\left(h_{r}+P \theta_{2}\right)\left(\frac{a+b t_{w}+c t_{w}^{2}}{\theta_{2}}\right)\left(e^{\theta_{2} t_{w}}-1\right)+\right.$

$\left(h_{0}+P \theta_{1}\right)\left\{w e^{\theta_{1} t_{w}}-\frac{e^{\theta_{1}\left(t_{w}-t\right)}}{\theta_{1}^{3}}\left[\theta_{1}^{2}(a+b T+\right.\right.$ $\left.\left.c T^{2}\right)-\theta_{1}(b+2 c)+2 c\right]+\frac{1}{\theta_{1}^{3}}\left[-\theta_{1}(b+c)+\right.$

$\left.\left.\left.\theta_{1}^{2}\left(a+b t_{w}+c t_{w}^{2}\right)+2 c\right]\right\}\right\}$

$$
\frac{\partial T C_{3}\left(t_{w}, T\right)}{\partial T}=0
$$

$\Rightarrow \frac{1}{T}\left\{\left(h_{0}+P \theta_{1}\right)\left\{\frac{a+b T+c T^{2}}{\theta_{1}} e^{\theta_{1}\left(T-t_{w}\right)}-\frac{b+2 c T}{\theta_{1}^{2}}+\frac{2 c}{\theta_{1}^{3}}\right\}+\right.$

$P I_{p}\left\{\frac{a+b T+c T^{2}}{\theta_{1}}\left(e^{\theta_{1}(T-M)}-1\right)\right\}+p_{1} I_{e}\left(a+b T+c T^{2}\right)-$

$\left.\frac{11 p_{1} I_{e}}{12}(6 a+4 b+3 c)\right\}-$

$\frac{1}{T^{2}}\left\{r+\left(h_{r}+P \theta_{2}\right)\left\{\frac{1-e^{\theta_{2}\left(t_{w}-t\right)}}{\theta_{2}^{4}}\left[\theta_{2}^{2}\left(a+b t_{w}+c t_{w}^{2}\right)-\right.\right.\right.$

$\left.\theta_{2}\left(b+2 c t_{w}\right)+2 c\right]+\frac{1}{\theta_{2}^{3}}\left[\theta_{2}\left(b t_{w}+c t_{w}^{2}\right)-\right.$

$\left.\left.\theta_{2}^{2}\left(a t_{w}+\frac{b t_{w}^{2}}{2}+\frac{c t_{w}^{3}}{3}\right)-2 c t_{w}\right]\right\}+\left(h_{0}+P \theta_{1}\right)\left\{\frac{w}{\theta_{1}}(1-\right.$

$e^{\left.-\theta_{1} t_{w}\right)}-\frac{1-e^{\theta_{1}\left(T-t_{w}\right)}}{\theta_{1}^{4}}\left[\theta_{1}^{2}\left(a+b T+c T^{2}\right)-\theta_{1}(b+\right.$

$2 c T)+2 c]+\frac{1}{\theta_{1}^{3}}\left[\theta_{1}\left[b\left(T-t_{w}\right)+c\left(T^{2}-t_{w}^{2}\right)\right]-\right.$

$\left.\left.\theta_{1}^{2}\left(a\left(t_{w}-T\right)+\frac{b\left(t_{w}^{2}-T^{2}\right)}{2}+\frac{c\left(t_{w}^{3}-T^{3}\right)}{3}\right)+2 c\left(t_{w}-T\right)\right]\right\}+$

$\mathrm{PI}_{\mathrm{p}}\left\{\frac{1}{\theta_{1}}\left[\left(\frac{a+b T+c T^{2}}{\theta_{1}}\right)\left(e^{\theta_{1}(T-M)}-1\right)+a(M-T)+\right.\right.$

$\left.\frac{b\left(M^{2}-T^{2}\right)}{2}+\frac{c\left(M^{3}-T^{3}\right)}{3}\right]+\frac{1}{\theta_{1}^{2}}\left[[b T-M)+c\left(T^{2}-M^{2}\right)\right]+$

$\left.\left.\left(\frac{b+2 c T}{\theta_{1}}\right)\left(1-e^{\theta_{1}(T-M)}\right)\right]+\frac{2 c}{\theta_{1}^{3}}\left[\frac{e^{\theta_{1}(T-M)}-1}{\theta_{1}}+(M-T)\right]\right\}-$

$\left.p_{1} I_{e}\left\{\frac{T^{2}(6 a+4 b+3 c)}{12}+T(M-T)\left(a+b T+c T^{2}\right)\right\}\right\}$

\subsection{Algorithm}

Step 1. Input the parameters.

Step 2. Solving Equations (16) and (17) and find the optimal values of $t_{w 1}$ and $T_{1}$. If $M \leq t_{w 1} \leq T$, set $t_{w}^{*}=t_{w 1}, T^{*}=$ $T_{1}$. Otherwise go to next step.
Step 3. Solving Equations (19) and (20) and find the optimal values of $t_{w 2}$ and $T_{2}$. If $t_{w 2} \leq M \leq T$, set $t_{w}^{*}=t_{w 2}, T^{*}=$ $T_{2}$. Otherwise go to next step.

Step 4. Solving Equations (22) and (23) and find the optimal values of $t_{w 3}$ and $T_{3}$. If $\quad M>T$, set $t_{w}^{*}=t_{w 3}, T^{*}=T_{3}$. Otherwise go to next step.

Step 5. Let $\left(t_{w}^{*}, T^{*}\right)=\arg \min \left(T C_{1}, T C_{2}, T C_{3}\right)$ and out put the optimal $t_{w}^{*}, T^{*}$.

\section{NUMERICAL EXAMPLE}

Given an inventory system with the following parameters: $\mathrm{a}=500, \mathrm{~b}=0.1, \mathrm{c}=0.2$, ho $=1$, $\mathrm{hr}=5, \mathrm{p}=10, \mathrm{p}_{1}=15, \mathrm{I}_{\mathrm{e}}=0.12, \mathrm{I}_{\mathrm{r}}=0.15$, $\theta_{1}=0.1, \theta_{2}=0.06$. Different values of $\mathrm{r}, \mathrm{w}$,

\begin{tabular}{|c|c|c|c|c|c|}
\hline $\mathbf{w}$ & $\mathbf{r}$ & $\mathbf{M}$ & $\overline{t_{w}^{*}}$ & $\boldsymbol{T}^{*}$ & $T C^{*}$ \\
\hline \multirow[t]{9}{*}{50} & 3000 & 0.1 & 0.1130 & 0.4049 & $7.0954 \times 10^{3}$ \\
\hline & & 0.15 & 0.1065 & 6.6475 & $5.2568 \times 10^{4}$ \\
\hline & & 0.6 & 0.1380 & 0.5556 & $3.8214 \times 10^{3}$ \\
\hline & 3500 & 0.1 & 0.1130 & 0.4466 & $9.7204 \times 10^{3}$ \\
\hline & & 0.15 & 0.1064 & 6.6891 & $5.2916 \times 10^{4}$ \\
\hline & & 0.6 & 0.1130 & 0.4513 & $8.6519 \times 10^{3}$ \\
\hline & 4000 & 0.1 & 0.1129 & 0.4883 & $1.1931 \times 10^{4}$ \\
\hline & & 0.15 & 0.1064 & 6.7308 & $5.3263 \times 10^{4}$ \\
\hline & & 0.6 & 0.1129 & 0.4935 & $1.0621 \times 10^{4}$ \\
\hline \multirow[t]{7}{*}{100} & 3000 & 0.1 & 0.2134 & 0.5342 & $6.3015 \times 10^{3}$ \\
\hline & & 0.15 & 0.1589 & 6.6479 & $5.1803 \times 10^{4}$ \\
\hline & & 0.6 & 0.2134 & 0.5559 & $6.1407 \times 10^{3}$ \\
\hline & 3500 & 0.1 & 0.1653 & 0.4467 & 733.30 \\
\hline & & 0.15 & 0.1653 & 0.4473 & 469.93 \\
\hline & & 0.6 & 0.1653 & 0.4515 & 242.28 \\
\hline & 4000 & 0.1 & 0.1652 & 0.4884 & $3.7060 \times 10^{4}$ \\
\hline
\end{tabular}
$\mathrm{M}$ and the computational values are given in following table. 


\section{$\begin{array}{llll}0.15 & 0.1652 & 0.4890 & 3.4311 \times 10^{3}\end{array}$ \\ $\begin{array}{llll}0.6 & 0.1652 & 0.4937 & 1.2572 \times 10^{4}\end{array}$}

\section{CONCLUSION}

This paper deals with an optimal inventory model for a deteriorating item with time dependent quadratic demand and delay in payment for two ware houses. The quadratic time dependent demand assumes the form of $D(t)=a+b t+c t^{2}, a \geq 0, b \neq 0, c \neq 0$, where there is a better representation of time. In the real market, when compared to linear and exponential demands the quadratic demand seems to be the best approach. Because in case of linear demand there is uniform change, and exponential demand leads to high rate of change, which is not found in reality. The quadratic demand is suitable for seasonal products, where the demand becomes high in the middle of the season and low towards the end. This type of demand is more realistic than the other two demand discussed here. In the proposed model, two warehouses (like RW, OW) are considered for storing goods. It was assumed that in rented warehouse the items have to be disposed earlier than the owned warehouse because of high cost incurred, even in spite of this the rent warehouse is preferred because of its storing and preserving facilities. Numerical examples are also provided to illustrate the proposed model.

The proposed model can further extended by taking more realistic assumptions such as finite rate of replenishment, stochastic demand patterns, quantity discounts, time value of money, multi products etc.

\section{REFERENCES}

[1] Aggarwal, S.P., and Jaggi, C.K., 1995. Ordering Policies of Deteriorating items under permissible delay in payments, Journal of the Operational Research Society, 46, 658-662.

[2] Backer, R.C., and Urban, L.A., 1988. Deterministic inventory system with an inventory level- dependent demand rate, Journal of the Operational Research Society, 39, 823-831.

[3] Biswajit Sarkar, 2012. An EOQ model with delay in payments and time varying deterioration rate, Mathematical and Computer Modelling, 55, 367-377.

[4] Chang, H. J., and Dye, C.Y., 1999. An EOQ model for deteriorating items with time varying demand and partial backlogging, Journal of the Operational Research Society, 50, 1176-1182.

[5] Chia- Huei Ho, 2011. The optimal integrated inventory policy with price-andcredit-linked demand under two-level trade credit, Computers and Industrial Engineering, 60, 117-126.

[6] Chung K.J., Huang T.S., 2007. The optimal retailer's ordering policies for deteriorating items with limited storage capacity under trade credit financing, International Journal of Production Economics, 106, 127-145.

[7] Dey J.K., Shyamal Kumar Mondal, Manoranjan Maiti, 2008. Two storage inventory problem with dynamic demand and interval valued lead-time over finite time horizon under inflation and time-value of money, European Journal of Operational Research, 185, 170-194.

[8] Goyal, S.K., 1985. Economic order quantity under conditions of permissible delay in payments, Journal of the Operational Research Society, 36, 335-338.

[9] Gupta, R., and Vrat, P., 1986. Inventory model for stock dependent consumption rate, Opsearch, 23, 19-24.

[10] Hwang, H., and Shrinn, S.W., 1997. Retailers pricing and lot sizing policy for exponentially deteriorating product under the condition of permissible delay in payments, Computers and Operations Research, 24, 539-547.

[11] Jamal, A.K., Sarker, B.R., and Wang, S., 1997. An ordering policy for deteriorating items with allowable shortage and 
permissible delay in payment, Journal of the Operational Research Society, 48, 826833.

[12] Lee, C.C., 2006. Two-warehouse inventory model with deterioration under FIFO dispatching policy, European Journal of Operational Research, 174, 861-873.

[13] Lee C.C., Hsu S.L., 2009. A twowarehouse production model for deteriorating inventory items with timedependent demands, European Journal of Operational Research, 194, 700-710.

[14] Mandal, B.N., and Phaujadar, S., 1989. An inventory model for deteriorating items and stock-dependent consumption rate, Journal of the Operational Research Society, 40, 483-488.

[15] Niu B., Xie J.X., 2008. A note on Twowarehouse inventory model with deterioration under FIFO dispatch policy, European Journal of Operational Research, 190, 571-577

[16] Padmanabhan, G., and Vrat, P., 1995. EOQ models for perishable items under stock dependent selling rate, European Journal of the Operational Research, 86, 281-292.

[17] Pakkala T.P.M., Achary K.K. , 1992. A deterministic inventory model for deteriorating items with two warehouses and finite replenishment rate, European Journal of Operational Research, $57,71-76$.

[18] Rong M., Mahapatra N.K., Maiti M., 2008. A two warehouse inventory model for a deteriorating item with partially/fully backlogged shortage and fuzzy lead time, European Journal of Operational Research, 189, 59-75.

[19] Saoussen Krichen, Awatef Laabidi, Fouad Ben Abdelaziz, 2011. Single supplier multiple cooperative retailers inventory model with quantity discount and permissible delay in payments, Computers and Industrial Engineering, 60, 164-172.
[20] Sarker, B.R., Mukherjee, S., and Balan, C.V., 1997. An order - level lot size inventory model with inventory level dependent demand and deterioration, International Journal of Production Economics, 48, 227-236.

[21] Sarma K.V.S., 1987. A deterministic order level inventory model for deteriorating items with two storage facilities, European Journal of Operational Research, 29, 70-73.

[22] Shib Sankar Sana, 2011. Price-sensitive demand for perishable items - an EOQ model, Applied Mathematics and Computations, 217, 6248-6259.

[23] Uthayakumar, R., Parvathi, P., 2006. A deterministic inventory model for deteriorating items with time-dependent demand, backlogged partially when delay in payments is permissible, International Journal of Mathematical Sciences 5, 201219.

[24] Yanlai Liang, Fangming Zhou, 2011. A two-warehouse inventory model for deteriorating items under conditionally permissible delay in payment, Applied Mathematical Modelling, 35, 2221-2231.

[25] Zhou Y.W., 2003. A multi-warehouse inventory model for items with timevarying demand and shortages, Computers and Operations Research, 30, 2115-2134.

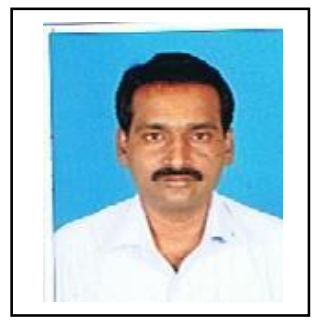

Mr. P. MUNIAPPAN is an Assistant Professor in the department of Mathematics, Sathyabama University, Chennai. He is doing $\mathrm{PhD}$ in the area of Inventory Management and Control with Supply Chain since 2013 in Sathyabama University, Chennai. $\mathrm{He}$ has published research articles in refereed journals and Conferences (National and International) in this area. 